\title{
Decrease of peripheral blood lymphocyte count predicts response to neoadjuvant chemotherapy in breast cancer patients
}

\author{
Ok Hee Lee, Sun-Young Min \\ Department of Surgery, Kyung Hee University College of Medicine, Seoul, Korea
}

Purpose: Pathologic complete response (pCR) after neoadjuvant chemotherapy (NAC) holds promise as a significant prognostic factor to predict NAC response in breast cancer patients. The absolute peripheral blood lymphocyte (PBL) count has been suggested as an independent predictor of response to NAC. The current study evaluated the relationship between $\mathrm{PCR}$ and the change of PBL count in patients treated with NAC.

Methods: A total of 61 patients with histologically confirmed breast cancer treated with NAC followed by mastectomy between January 2010 and December 2019 were analyzed retrospectively. Correlational analyses confirmed a statistically significant relationship between PBL count and pCR. Following conformational correlational analyses, patients were divided into two groups according to cutoff values using the receiver operating characteristics curve and a logistic regression was conducted to determine the optimal conditions for achieving $\mathrm{pCR}$.

Results: A total of 14 patients (22.9\%) achieved pCR. Most PBL counts decreased after NAC relevant to pCR. Logistic regression analysis revealed that a small decrease of $P B L$ was associated with $p C R(P=0.028)$. The cutoff value of $P B L$ decrease was $755 \times 10^{6} / L$, which was used to divide patients into high and low reduction groups. The PCR rate was $11.43 \%$ and $38.46 \%$ for the high and low reduction group, respectively (area under the curve, $0.707 ; 95 \%$ confidence interval, $0.556-0.858 ; \mathrm{P}=0.020$ ). The high reduction group was found to have more difficulty achieving $\mathrm{pCR}$.

Conclusion: The decrease of PBL is significantly associated with $\mathrm{pCR}$. Our data support that the decrease of PBL after NAC may be useful factors in predicting the response to NAC in breast cancer patients.

Keywords: Breast neoplasms, Neoadjuvant chemotherapy, Pathological complete remission, Peripheral blood lymphocytes

\section{INTRODUCTION}

Neoadjuvant chemotherapy (NAC) is currently recommended as the treatment of choice for patients with locally advanced breast cancer and large sized non-operable tumors. NAC is usually recommended for patients who are expected to experience poor cos-

Received: Sep 21, 2020 Revised: Nov 12, 2020 Accepted: Dec 2, 2020

Correspondence to: Sun-Young Min

Department of Surgery, Kyung Hee University College of Medicine,

23 Kyungheedae-ro, Dongdaemun-gu, Seoul 02447, Korea

Tel: +82-2-958-8375, Fax: +82-2-966-9366

E-mail: breastdrmin@gmail.com

ORCID: Ok Hee Lee (https://orcid.org/0000-0002-1385-5185), Sun-Young Min (https://orcid.org/0000-0003-2912-1167)

Copyright (C) 2020 Korean Society of Surgical Oncology

This is an Open Access article distributed under the terms of the Creative Commons Attribution Non-Commercial License (http://creativecommons.org/licenses/by-nc/4.0) which permits unrestricted non-commercial use, distribution, and reproduction in any medium, provided the original work is properly cited. metic outcomes after surgery due to large tumor size [1]. Recently, pathologic complete response (CR) after NAC has been reported as a good prognostic factor for predicting long-term outcomes such as disease-free survival (DFS) and overall survival (OS) in breast cancer patients $[2,3]$. These findings highlight a need to determine which factors predict better responses and which factors lead to a pathologic CR and improved long-term outcome.

To date, the prediction of pathologic CR is based on histologic and biologic subtype at diagnosis, the administered NAC regimen, and breast imaging results. Prior to surgery, clinical assessment was conducted on primary tumor to evaluate the effect of the chemotherapy regimen 3 weeks after completion of NAC. Physical examination, breast ultrasound, mammography and breast magnetic resonance imaging (MRI) may be used for assessment of the clinical CR [4]. Clinical CR often could be used as a surrogate marker for evaluation of the efficacy of NAC before assessment of the pathological response. However, clinical CR is just one of the predictive factors and does not determine pathologic CR directly. 
Gazet et al. [5] revealed that in those patients with a complete CR, $84 \%$ of patients had evidence of residual tumor on histological examination. Surgical excision is the gold standard in determining NAC response rate.

Previous studies have described that a higher rate of pathologic CR is associated with several subtype factors: neoadjuvant regimens such as anthracycline and taxane, hormone receptor status, an expression of human epidermal growth factor receptor-2 (HER-2) and Ki-67 and other mutant genes, and histologic type $[6,7]$. These factors have a certain correlation with pathologic CR; however, are not easily used as predictive markers in clinical practice. Therefore, more intuitive and convenient indicators are needed.

In patients with breast cancer, the immune response is complex and plays an important role in cancer progression. Some observations suggest that chemotherapy may trigger an immune response against a tumor, contributing to treatment response. In previous studies, many researchers tried to assess the role of peripheral blood lymphocyte (PBL) count in cancer prognosis as peripheral blood cell counts were considered to reflect the immune status of cancer patients treated with chemotherapy [8]. Some studies have shown an association between tumor-associated lymphocytes and chemotherapy response in various solid tumors. According to recent data, sustaining PBL count during preoperative chemoradiotherapy was associated with a better pathologic CR rate in locally advanced rectal cancer [9]. A study investigating advanced gastric cancer found that high circulating lymphocyte ratios before and after NAC were positively associated with pathologic CR, suggesting its possible use as a new prognostic biomarker [10]. Similarly, a retrospective cohort study reported a positive relationship between pre-treatment PBL level and chemotherapy response, suggesting it is not only prognostic but has potential predictive value in breast cancer as well [8].

Taken together, these findings raise the question of which treatments may have an impact on the response to NAC and which immune components in blood are valuable as indicators to predict therapeutic effects. There are few reports that clarify an association between PBL and outcomes in breast cancer [11-13]. In the current study, we analyzed the relationship between pathologic CR and changes of PBL counts during NAC to determine what, if any, prognostic value it may have.

\section{METHODS}

\section{Patients}

Clinical data were retrospectively collected from 575 patients diagnosed with primary breast cancer through biopsy between January
2010 and December 2019 at Kyung Hee Medical Center in Seoul. Of the total 575 patients, 61 without distant metastases who received surgical resection after NAC were selected for review. Patients were evaluated with physical examination, routine blood tests, chest X-ray, mammography, ultrasound breast exam, breast MRI, and core needle biopsy of the primary tumor. Pre-NAC nodal status was determined by axillary and/or supraclavicular ultrasound-guided fine-needle aspiration. When evaluation of nodal metastasis was negative, a sentinel lymph node biopsy was performed. In locally advanced tumors, positron emission tomography-computed tomography (PET-CT), chest and abdominal CT were added to determine tumor stage. Clinicopathological characteristics included age, body mass index (BMI), menopausal status, tumor size, hormone receptors, HER-2 expression status, biologic subtype, chemotherapy regimen, and type of surgery. Written informed consent was obtained from all patients. The study was approved by the Ethics Committee of Kyung Hee Medical Center (IRB No. 2020-12-102-001).

Patients were excluded from the study based on diagnosis of bilateral breast cancer, metastatic stage, presence of chronic diseases such as chronic hepatic disease, chronic renal failure, and systemic inflammatory disease. Patients without assessable staging were excluded from the study as well.

\section{Immunohistologic characteristics, TNM grade}

Expression of estrogen receptor (ER) and progesterone receptor (PR) were defined using the Allred scoring system and assessed by immunohistochemistry. HER-2 was considered positive by immunohistochemistry for 3+, or if HER-2 status was at least $2+$ undergoing fluorescence in situ hybridization test.

Intrinsic subtypes were classified as follows: luminal A (ER positive, PR positive, HER-2 negative, and Ki-67 < 14\%); luminal B (ER positive, PR positive or negative, HER-2 positive or negative, Ki-67 $>14 \%$ ); HER-2 overexpression (ER negative, PR negative, HER-2 positive, independently of Ki-67 value); and triple negative (ER negative, PR negative, HER-2 negative, independently of Ki-67 value).

Tumor stage was stratified based on the TNM classification according to The American Joint Committee on Cancer 8th edition [14].

\section{Neoadjuvant chemotherapy treatment}

Patients receiving neoadjuvant therapy of anthracycline and/or taxanes sequential regimens were included in the current study. Patients with HER-2 overexpression type were treated with trastuzumab. The most common regimen used was; doxorubicin $(60$ $\mathrm{mg} / \mathrm{m}^{2}$ for 4 cycles) and cyclophosphamide $\left(600 \mathrm{mg} / \mathrm{m}^{2}\right.$ with 3 
weeks interval for 4 cycles), followed with paclitaxel $\left(80 \mathrm{mg} / \mathrm{m}^{2}\right.$ weekly for 12 cycles) or docetaxel $\left(75 \mathrm{mg} / \mathrm{m}^{2}\right.$ with 3 weeks interval for 4 cycles). Patients were divided into two groups; target agent group and non-target agent group according to the use of trastuzumab.

\section{Radiographic and pathological assessment}

To evaluated tumor response, a breast MRI was performed before and after NAC or prior to surgery. A pathologic CR was defined as the absence of invasive carcinoma or ductal carcinoma in situ in breast and axilla after operation (ypT0ypN0).

\section{Peripheral blood lymphocytes assessment}

Blood tests were routinely performed during each cycle and after NAC. The count of lymphocyte, neutrophil, and platelets were examined from data available at the Hospital's laboratory database. Due to the retrospective design of the current study, the timing of blood tests were different for each patient. All data was plotted just prior to the initiation of NAC.

The normal range of absolute lymphocyte count was 1,000$4,800 \times 10^{6} / \mathrm{L}$. The number of lymphocytes were determined in peripheral blood at baseline, after the 1st cycle of chemotherapy again after completion of chemotherapy (typically 3 weeks after the last cycle).

\section{Statistical analyses}

The main purpose of this study was to assess the role of PBL changes during neoadjuvant therapy as a potential predictive marker of pathologic CR in breast cancer patients. Clinicopathologic parameters were compared between the pathologic CR and non-pathologic CR groups using chi-square tests or Fisher exact test. The Wilcoxon rank-sum test was performed to see if pathologic CR improved with changes in PBL count. According to receiver operating characteristics (ROC) studies, an optimal cutoff value was established to discriminate between the two groups: low PBL reduction group $\left(<755 \times 10^{6} / \mathrm{L}\right)$ and high PBL reduction group $\left(\geq 755 \times 10^{6} / \mathrm{L}\right)$. In addition, we used Fisher exact test to determine which variable was significantly associated with pathologic CR. Attempts were made to find valid factors that could affect pathologic CR and determine whether the change of PBL count is an independent factor. Finally, significant variables related to pathologic CR were identified in logistic regression using a "stepwise" selection method. Analyses included Firth logistic regression, a penalized likelihood method which is used to reduce small-sample bias in maximum likelihood estimation. Statistical significance was defined as P-values less than 0.05. Statistical analysis was carried out using SAS 9.4 (SAS Institute Inc., Cary, NC,
USA) and SPSS 23 (IBM Corp., Armonk, NY, USA).

\section{RESULTS}

A total of 61 patients were included in this study. Clinicopathologic characteristics of the patients are shown in Table 1. The median age of patients was 50.5 years ( $50.57 \pm 8.80$; interquartile range, 50 ; range $34-75$ years old). Approximately $68.8 \%$ of the patients were in a postmenopausal state. Over half of the patients had a BMI of $20-25 \mathrm{~kg} / \mathrm{m}^{2}$. All tumors were invasive ductal carcinoma and locally advanced in $53(86.8 \%)$ patients (24.5\% of IIB, $49.1 \%$ of IIIA, $9.8 \%$ of IIIB, and $3.2 \%$ of IIIC). Most patients, 56 of 61 (91.8\%), presented with compromised lymph nodes (N1-N3). In our cohort, the most frequent tumor molecular subtype was luminal B (34.4\%), with other subtypes distributed as follows: $27.8 \%$ of luminal A, $19.6 \%$ of triple negative, and $18 \%$ of HER-2 enriched. Neoadjuvant target therapy using trastuzumab occurred in $27.8 \%$ of patients. The most used chemotherapy regimen was sequential anthracyclines and taxanes in $67.2 \%$. Overall pathologic CR rate was $22.9 \%$. Partial mastectomy was performed in $60.6 \%$ and axillary lymph node dissection was performed in $52.4 \%$ of cases.

In this study, 14 patients (22.9\%) achieved pathologic CR. Results of analysis of tumor and treatment-related characteristics with pathologic CR are reported in Table 2. Patients who achieved pathologic CR tended to be typically in a premenopausal state, with low tumor grade (I and II), luminal B type, and had received trastuzumab therapy. Significant correlations were found between these factors and pathologic CR. Patients not treated with trastuzumab had a somewhat lower pathologic CR rate compared to those with treated with trastuzumab. It was observed that patients who received neoadjuvant target chemotherapy achieved pathologic CR more often than patients who received only anthracycline and/or taxanes sequential regimens. Differences in HER-2 overexpression state were not statistically significant. The tumor subtype with the highest response rates was luminal B (57.1\%), followed by triple-negative type (28.5\%).

As shown in Table 3, the mean baseline PBL was $1,821 \times 10^{6} / \mathrm{L}$ (range, 697-3,281 $\times 10^{6} / \mathrm{L}$; standard deviation, $550.45 \times 10^{6} / \mathrm{L}$ ). Interestingly, there was no association between initial PBL level and pathologic $\mathrm{CR}(\mathrm{P}=0.600)$. In the initial status, lymphocytopenia (defined as $\mathrm{PBL}<950 \times 10^{6} / \mathrm{L}$ ) was observed in $3.2 \%$ of patients and its presence was not associated with pathologic CR. Differences in PBL counts after 1st cycle of NAC were not statically significant $(\mathrm{P}=0.879)$. However, there was a statistically significant smaller change and larger absolute level of PBL counts after NAC associated with a higher rate of pathologic $\mathrm{CR}(\mathrm{P}=0.019$ and $\mathrm{P}=0.019$, respectively). There was no significant difference in 
Table 1. Clinicopathological characteristics of breast cancer patients with neoadjuvant chemotherapy

\begin{tabular}{|c|c|}
\hline Characteristics & No. of patients $(\%)$ \\
\hline \multicolumn{2}{|l|}{ Age (yr) } \\
\hline Mean $\pm S D$ & $50.5 \pm 8.8$ \\
\hline Median (IOR) & $50(44-56)$ \\
\hline \multicolumn{2}{|l|}{ Age group (yr) } \\
\hline$<35$ & $1(1.6)$ \\
\hline $35-44$ & $15(25.0)$ \\
\hline $45-54$ & $28(46.6)$ \\
\hline $55-64$ & $12(20.0)$ \\
\hline $65-75$ & $5(8.1)$ \\
\hline \multicolumn{2}{|l|}{ Menopausal status } \\
\hline Premenopausal & $19(31.1)$ \\
\hline Postmenopausal & $42(68.8)$ \\
\hline \multicolumn{2}{|l|}{ BMI $\left(\mathrm{kg} / \mathrm{m}^{2}\right)$} \\
\hline$<20$ & $2(3.2)$ \\
\hline 20 to $<25$ & $36(59.0)$ \\
\hline 25 to $<30$ & $19(31.1)$ \\
\hline$>30$ & $4(6.5)$ \\
\hline \multicolumn{2}{|l|}{ Tumor grade } \\
\hline $\mathrm{T} 1$ & $9(14.7)$ \\
\hline T2 & $37(60.6)$ \\
\hline T3 & $8(13.1)$ \\
\hline $\mathrm{T} 4$ & $7(11.4)$ \\
\hline \multicolumn{2}{|l|}{ Lymph node } \\
\hline No & $5(8.2)$ \\
\hline N1 & 24 (39.3) \\
\hline N2 & $29(47.5)$ \\
\hline N3 & $3(4.9)$ \\
\hline \multicolumn{2}{|l|}{ Clinical staging } \\
\hline$\|$ & $23(37.7)$ \\
\hline III & $38(62.3)$ \\
\hline \multicolumn{2}{|l|}{ ER state } \\
\hline Positive & 38 (62.3) \\
\hline Negative & $23(37.7)$ \\
\hline \multicolumn{2}{|l|}{ PR state } \\
\hline Positive & $33(54.1)$ \\
\hline Negative & $28(45.9)$ \\
\hline \multicolumn{2}{|l|}{ HER-2 overexpression } \\
\hline Positive & 25 (40.9) \\
\hline Negative & $36(59.0)$ \\
\hline \multicolumn{2}{|l|}{ Biological type } \\
\hline Luminal A & $17(27.8)$ \\
\hline Luminal B & $21(34.4)$ \\
\hline HER-2 enriched & $11(18.0)$ \\
\hline Triple negative & $12(19.6)$ \\
\hline \multicolumn{2}{|c|}{ Neoadjuvant chemotherapy regimen } \\
\hline Non-target regimen & $44(72.1)$ \\
\hline Target regimen & $17(27.8)$ \\
\hline
\end{tabular}

Table 1. Continued

\begin{tabular}{lc}
\hline Characteristics & No. of patients (\%) \\
\hline Pathological response & \\
Pathologic CR & $14(22.9)$ \\
No pathologic CR & $47(77.0)$ \\
Operation & \\
PM & $1(1.6)$ \\
PM with SNBx & $6(9.8)$ \\
PM with ALND & $30(49.1)$ \\
TM with SNBx & $22(36.0)$ \\
MRM & $2(3.2)$ \\
\hline
\end{tabular}

SD, standard deviation; IQR, interquartile range; $B M I$, body mass index; $E R$, estrogen receptor; $\mathrm{PR}$, progesterone receptor; HER-2, human epidermal growth factor receptor; $C R$, complete response; $P M$, partial mastectomy; TM, total mastectomy; MRM, modified radical mastectomy; SNBx, sentinel node biopsy; ALND, axillary lymph node dissection.

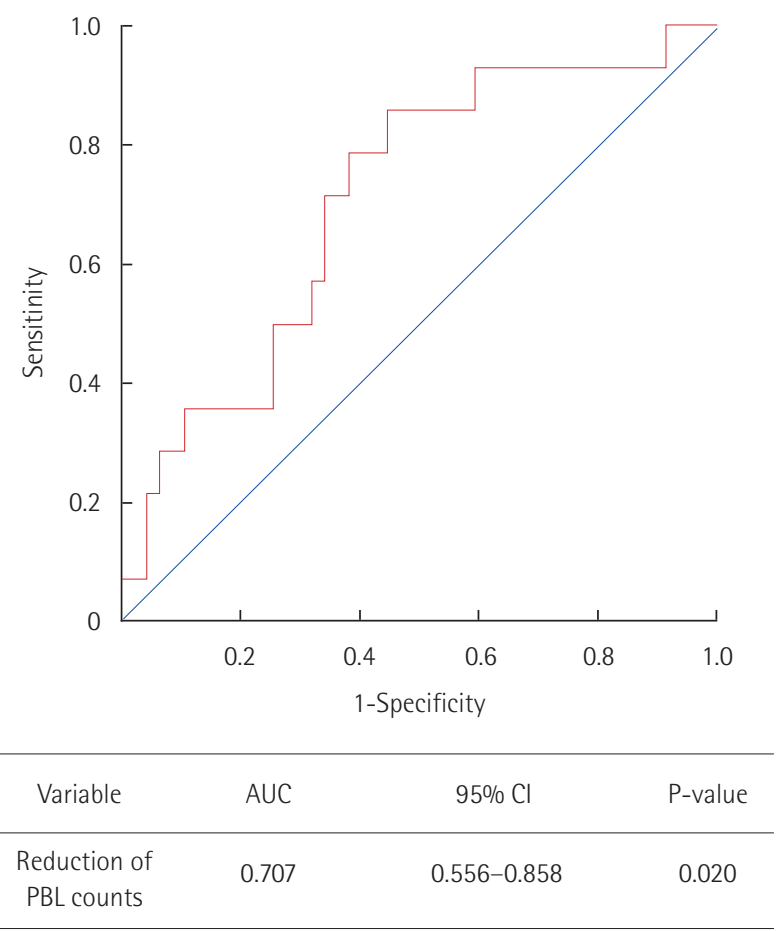

Fig. 1. Receiver operating characteristics curve (ROC) analysis for PBL reduction. PBL, peripheral blood lymphocyte; AUC, area under the curve; $\mathrm{Cl}$, confidence interval.

platelet and neutrophil counts with pathologic CR at any time point during NAC.

ROC curve analysis proposed an optimal cutoff value of PBL reduction for pathologic CR achieving group in Fig. 1. The calculated cutoff point of decreased PBL counts was $755 \times 10^{6} / \mathrm{L}$ with $71.43 \%$ of sensitivity and $65.96 \%$ of specificity. Using this cutoff point, patients were grouped by high reduction (PLR $<755 \times 10^{6} \%$ 
Table 2. Associations between pathologic CR and clinicopathological characteristics of breast cancer patients

\begin{tabular}{|c|c|c|c|}
\hline Characteristics & $\begin{array}{c}\text { Pathologic CR } \\
\text { negative, No. (\%) }\end{array}$ & $\begin{array}{c}\text { Pathologic CR } \\
\text { positive, No. (\%) }\end{array}$ & P-value ${ }^{a)}$ \\
\hline No. of patients & 47 & 14 & \\
\hline Age (yr) & & & $0.077^{b)}$ \\
\hline Mean \pm SD & $51.6 \pm 8.6$ & $46.9 \pm 8.4$ & \\
\hline Median (IQR) & $51.0(45.5-56.5)$ & $44.5(39.5-49.5)$ & \\
\hline Menopausal status & & & 0.024 \\
\hline Premenopausal & $11(23.4)$ & $8(57.1)$ & \\
\hline Postmenopausal & $36(76.6)$ & $6(42.8)$ & \\
\hline $\mathrm{BMI}\left(\mathrm{kg} / \mathrm{m}^{2}\right)$ & & & 1.000 \\
\hline$<20$ & $2(4.2)$ & 0 & \\
\hline 20 to $<25$ & $27(57.4)$ & $9(64.2)$ & \\
\hline 25 to $<30$ & $15(31.9)$ & $4(28.5)$ & \\
\hline$>30$ & $3(6.3)$ & $1(7.1)$ & \\
\hline Tumor grade & & & 0.053 \\
\hline $\mathrm{T} 1$ & $4(8.5)$ & $5(35.7)$ & \\
\hline $\mathrm{T} 2$ & $30(63.8)$ & $7(50)$ & \\
\hline $\mathrm{T} 3$ & $6(12.7)$ & $2(14.2)$ & \\
\hline $\mathrm{T} 4$ & $7(14.8)$ & 0 & \\
\hline Lymph node & & & 0.759 \\
\hline No & $3(6.3)$ & $2(14.2)$ & \\
\hline N1 & $19(40.4)$ & $5(35.7)$ & \\
\hline N2 & $22(46.8)$ & $7(50)$ & \\
\hline N3 & $3(6.3)$ & 0 & \\
\hline Clinical staging & & & 0.650 \\
\hline$\|$ & $17(36.1)$ & $6(42.8)$ & \\
\hline III & $30(63.8)$ & $8(57.1)$ & \\
\hline ER state & & & 1.000 \\
\hline Positive & $29(61.7)$ & $9(64.2)$ & \\
\hline Negative & 18 (38.3) & $5(35.7)$ & \\
\hline PR state & & & 0.374 \\
\hline Positive & $27(57.4)$ & $6(42.8)$ & \\
\hline Negative & $20(42.5)$ & $8(57.1)$ & \\
\hline HER-2 overexpression & & & 0.218 \\
\hline Positive & $17(36.1)$ & $8(57.1)$ & \\
\hline Negative & $30(63.8)$ & $6(42.8)$ & \\
\hline Biological type & & & 0.056 \\
\hline Luminal A & $16(34.0)$ & $1(7.1)$ & \\
\hline Luminal B & $13(27.6)$ & $8(57.1)$ & \\
\hline HER-2 enriched & $10(21.2)$ & $1(7.1)$ & \\
\hline Triple negative & $8(17.0)$ & $4(28.5)$ & \\
\hline \multicolumn{3}{|c|}{ Neoadjuvant chemotherapy regimen } & 0.047 \\
\hline Non-target regimen & $37(78.7)$ & $7(50.0)$ & \\
\hline Target regimen & $10(21.2)$ & $7(50.0)$ & \\
\hline
\end{tabular}

$\mathrm{CR}$, complete response; $\mathrm{SD}$, standard deviation; IQR, interquartile range; $\mathrm{BMI}$, body mass index; ER, estrogen receptor; PR, progesterone receptor; HER-2, human epidermal growth factor receptor.

a) Fisher exact test. ${ }^{b}$ Independent t-test.
Table 3. Associations between pathologic complete response and variables of blood components

\begin{tabular}{|c|c|c|c|}
\hline Characteristics & $\begin{array}{c}\text { Pathologic } \mathrm{CR} \\
\text { negative }\end{array}$ & $\begin{array}{c}\text { Pathologic CR } \\
\text { positive }\end{array}$ & P-value ${ }^{a)}$ \\
\hline No. of patients & 47 & 14 & \\
\hline \multicolumn{4}{|l|}{$\mathrm{A}\left(\times 10^{6} / \mathrm{L}\right)$} \\
\hline Lymphocyte & $1,841.8 \pm 570.5$ & $1,753.0 \pm 489.8$ & 0.600 \\
\hline Neutrophil & $60.0 \pm 6.5$ & $59.6 \pm 14.7$ & 0.835 \\
\hline Platelet & $274.6 \pm 54.7$ & $272.8 \pm 58.0$ & 0.917 \\
\hline \multicolumn{4}{|l|}{$B\left(\times 10^{6} / L\right)$} \\
\hline Lymphocyte & $1,076.4 \pm 473.2$ & $1,056.0 \pm 287.4$ & 0.879 \\
\hline Neutrophil & $49.6 \pm 23.5$ & $53.0 \pm 28.7$ & $0.511^{b)}$ \\
\hline Platelet & $226.0 \pm 77.8$ & $215.2 \pm 69.6$ & $0.871^{b)}$ \\
\hline \multicolumn{4}{|l|}{$C\left(\times 10^{6} / L\right)$} \\
\hline Lymphocyte & $984.2 \pm 376.8$ & $1,274.9 \pm 457.7$ & 0.019 \\
\hline Neutrophil & $58.3 \pm 17.1$ & $55.1 \pm 14.4$ & $0.585^{b)}$ \\
\hline Platelet & $283.4 \pm 77.6$ & $297.7 \pm 109.9$ & 0.586 \\
\hline \multicolumn{4}{|l|}{$\Delta_{D}\left(\times 10^{6} / L\right)$} \\
\hline Lymphocyte & $-765.3 \pm 537.3$ & $-697.0 \pm 596.5$ & 0.685 \\
\hline Neutrophil & $-10.8 \pm 22.7$ & $-6.0 \pm 30.2$ & $0.626^{b)}$ \\
\hline Platelet & $-48.5 \pm 82.5$ & $-57.5 \pm 67.2$ & $0.688^{b)}$ \\
\hline \multicolumn{4}{|l|}{$\Delta_{E}\left(\times 10^{6} / L\right)$} \\
\hline Lymphocyte & $-857.5 \pm 492.1$ & $-478.1 \pm 604.0$ & 0.019 \\
\hline Neutrophil & $-2.2 \pm 18.4$ & $-4.5 \pm 17.8$ & $0.700^{b)}$ \\
\hline Platelet & $8.8 \pm 75.1$ & $24.8 \pm 73.0$ & 0.483 \\
\hline
\end{tabular}

Values are presented as mean \pm standard deviation.

$C R$, complete response; $A$, before the initiation of neoadjuvant chemotherapy;

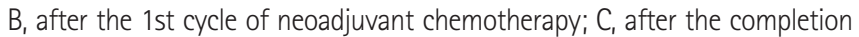
of neoadjuvant chemotherapy; $\Delta_{D_{1}}$ changes in blood components count after the 1st cycle of neoadjuvant chemotherapy; $\Delta_{E_{1}}$ changes in blood components count after completion of neoadjuvant chemotherapy.

${ }^{a}$ Independent t-test. ${ }^{b)}$ Wilcoxon rank-sum test.

$\mathrm{L}$ ) and low reduction (PLR $\geq 755 \times 10^{6} / \mathrm{L}$ ), accordingly. Changes in PBL count during NAC ranged between $-2,042$ and $937 \times 10^{6} / \mathrm{L}$ (mean, $-770.49 \times 10^{6} / \mathrm{L}$; median, $-813 \times 10^{6} / \mathrm{L}$; SD, $539.10 \times 10^{6} / \mathrm{L}$ ). When comparing groups, pathologic CR was significantly higher in the low decrease PBL group compared with the high group (area under the curve, $0.707 ; 95 \%$ confidence interval [CI], 0.556-0.858, $\mathrm{P}=0.020$ ). Of the total 14 patients with pathologic $\mathrm{CR}, 10$ patients were in the low reduction group and the other four patients were in the high reduction group.

Table 4 compares the characteristics between the low reduction group (PLR $\geq 755 \times 10^{6} / \mathrm{L}$ ) and the high reduction group (PLR $<755 \times 10^{6} / \mathrm{L}$ ). Hormone receptor state including ER, PR, and HER-2 overexpression state were not significantly correlated with PBL count reduction. As shown in the Table 3, analysis also demonstrated that the smaller decrease of PBL is related to better pathologic CR. In addition, there was a small decrease of PBL in 
Table 4. Clinicopathological characteristics in relation to reduction of PBL counts

\begin{tabular}{|c|c|c|c|}
\hline \multirow{2}{*}{ Characteristics } & \multicolumn{2}{|c|}{ Reduction of PBL counts } & \multirow{2}{*}{ P-value } \\
\hline & $<755 \times 10^{6} / \mathrm{L}$ & $\geq 755 \times 10^{6} / \mathrm{L}$ & \\
\hline No. of patients & 35 & 26 & \\
\hline Age (yr) & & & $0.143^{b)}$ \\
\hline Mean \pm SD & $52.00 \pm 8.90$ & $48.6 \pm 8.4$ & \\
\hline Median (IOR) & $51.0(44.5-57.5)$ & $49.0(43.5-54.5)$ & \\
\hline Age group (yr) & & & 0.550 \\
\hline$<35$ & $1(2.9)$ & 0 & \\
\hline $35-44$ & $6(17.6)$ & $9(34.6)$ & \\
\hline $45-54$ & $17(50.0)$ & $11(42.3)$ & \\
\hline $55-64$ & $8(23.5)$ & $4(15.3)$ & \\
\hline $65-75$ & $3(8.6)$ & $2(7.6)$ & \\
\hline Menopausal status & & & 0.161 \\
\hline Premenopausal & $8(22.8)$ & $11(42.3)$ & \\
\hline Postmenopausal & $27(77.1)$ & $15(57.6)$ & \\
\hline $\mathrm{BMI}\left(\mathrm{kg} / \mathrm{m}^{2}\right)$ & & & 0.040 \\
\hline$<20$ & $2(5.7)$ & 0 & \\
\hline 20 to $<25$ & $16(45.7)$ & $20(76.9)$ & \\
\hline 25 to $<30$ & $13(37.1)$ & $6(23.0)$ & \\
\hline$>30$ & $4(11.4)$ & 0 & \\
\hline Tumor grade & & & 0.764 \\
\hline $\mathrm{T} 1$ & $4(11.4)$ & $5(19.2)$ & \\
\hline T2 & $21(60.0)$ & $16(61.5)$ & \\
\hline T3 & $5(14.2)$ & $3(11.5)$ & \\
\hline $\mathrm{T} 4$ & $5(14.2)$ & $2(7.6)$ & \\
\hline Lymph node & & & 0.223 \\
\hline NO & $2(5.7)$ & $3(11.5)$ & \\
\hline N1 & $16(45.7)$ & $8(30.7)$ & \\
\hline N2 & $14(40.0)$ & $15(57.6)$ & \\
\hline N3 & $3(8.5)$ & 0 & \\
\hline Clinical staging & & & 0.667 \\
\hline$\|$ & $14(40.0)$ & $9(34.6)$ & \\
\hline III & $21(60.0)$ & $17(65.3)$ & \\
\hline ER state & & & 0.291 \\
\hline Positive & $24(68.5)$ & $14(53.8)$ & \\
\hline Negative & $11(31.4)$ & $12(46.1)$ & \\
\hline PR state & & & 0.127 \\
\hline Positive & $22(62.8)$ & $11(42.3)$ & \\
\hline Negative & $13(37.1)$ & $15(57.6)$ & \\
\hline HER-2 overexpression & & & 1.000 \\
\hline Positive & $14(40.0)$ & $11(42.3)$ & \\
\hline Negative & $21(60.0)$ & $15(57.6)$ & \\
\hline Biological type & & & 0.009 \\
\hline Luminal A & $14(40.0)$ & $3(11.5)$ & \\
\hline Luminal B & $10(28.5)$ & $11(42.3)$ & \\
\hline HER-2 enriched & 8 (22.8) & $3(11.5)$ & \\
\hline Triple negative & $3(8.5)$ & $9(34.6)$ & \\
\hline
\end{tabular}

Table 4. Continued

\begin{tabular}{|c|c|c|c|}
\hline \multirow{2}{*}{ Characteristics } & \multicolumn{2}{|c|}{ Reduction of PBL counts } & \multirow{2}{*}{$\mathrm{P}$-value ${ }^{\mathrm{a})}$} \\
\hline & $<755 \times 10^{6} / \mathrm{L}$ & $\geq 755 \times 10^{6} / \mathrm{L}$ & \\
\hline \multicolumn{4}{|c|}{ Neoadjuvant chemotherapy regimen } \\
\hline Non-target regimen & $25(71.4)$ & $19(73.0)$ & 1.000 \\
\hline Target regimen & $10(28.5)$ & $7(26.9)$ & \\
\hline \multicolumn{4}{|l|}{ Pathological response } \\
\hline Pathologic CR & $4(11.4)$ & $10(38.4)$ & 0.028 \\
\hline No pathologic CR & $31(88.5)$ & $16(61.5)$ & \\
\hline \multicolumn{4}{|l|}{ Operation } \\
\hline PM & $1(2.8)$ & 0 & 0.005 \\
\hline PM with SNBx & $4(11.4)$ & $2(7.6)$ & \\
\hline PM with ALND & $11(31.4)$ & $19(73.0)$ & \\
\hline TM with SNBx & $18(51.4)$ & $4(15.3)$ & \\
\hline MRM & $1(2.8)$ & $1(3.8)$ & \\
\hline
\end{tabular}

Values are presented as number (\%).

$P B L$, peripheral blood lymphocyte; $S D$, standard deviation; IQR, interquartile range; $B M I$, body mass index; $E R$, estrogen receptor; $P R$, progesterone receptor; HER-2, human epidermal growth factor receptor; $C R$, complete response; $\mathrm{PM}$, partial mastectomy; TM, total mastectomy; MRM, modified radical mastectomy; SNBx, sentinel node biopsy; ALND, axillary lymph node dissection.

${ }^{a)}$ Fisher exact test. ${ }^{b)}$ Independent t-test.

the luminal B group, suggesting that the luminal B group may have better response to NAC. To sum up, the results suggested that breast cancer patients with low reduction of PBL during NAC achieved a more favorable outcome, independent of primary tumor bio-molecular subtype.

The predictive value of each variable was compared by univariate analysis including PBL reduction and was statistically significant. Multivariate analysis indicated that only non-target agent and high PBL reduction were statistically significant. Compared to the high PBL reduction group, the low reduction group was seven times more likely to be pathologic CR positive (odds ratio [OR], 7.12; 95\% CI, 1.36-37.29; $\mathrm{P}=0.020$ ). In addition, target agent group using trastuzumab was 37 times more responsive to NAC compared to non-target agent group as reported in Tables 5 and 6 (OR, 37.86; 95\% CI, 3.63-394.59; P = 0.002).

\section{DISCUSSION}

NAC has been administered to locally advanced breast cancer patients more recently. Sometimes pathologic CR is proposed as a clinical endpoint for DFS and OS. Therefore, various analyses were conducted to discover a more sensitive tool for evaluating CR to NAC. Only clinical examination to detect residual disease or chemotherapy response is known to be limited. Prior retrospective analysis revealed that radiotherapy alone after NAC achieved sur- 
Table 5. Univariable analysis for predictive factors of pathologic complete response

\begin{tabular}{|c|c|c|c|}
\hline \multirow{2}{*}{ Characteristics } & \multicolumn{2}{|c|}{ Simple logistic regression } & \multirow{2}{*}{ P-value } \\
\hline & $\mathrm{OR}$ & $95 \% \mathrm{Cl}$ & \\
\hline \multicolumn{4}{|l|}{ Age group (yr) } \\
\hline$<35$ & 1.00 & & \\
\hline $35-44$ & 0.37 & $0.00-39.10$ & $0.678^{a)}$ \\
\hline $45-54$ & 1.80 & $0.02-188.21$ & $0.804^{\mathrm{a})}$ \\
\hline $55-64$ & 1.39 & $0.01-161.72$ & $0.892^{\mathrm{a})}$ \\
\hline $65-75$ & 0.77 & $0.01-116.17$ & $0.918^{\text {a) }}$ \\
\hline \multicolumn{4}{|l|}{ Menopausal status } \\
\hline Premenopausal & 1.00 & & \\
\hline Postmenopausal & 4.36 & $1.24-15.32$ & 0.021 \\
\hline \multicolumn{4}{|l|}{$\mathrm{BMI}\left(\mathrm{kg} / \mathrm{m}^{2}\right)$} \\
\hline$<20$ & 2.14 & $0.03-156.54$ & $0.727^{\mathrm{a})}$ \\
\hline 20 to $<25$ & 1.24 & $0.13-11.95$ & $0.852^{\mathrm{a})}$ \\
\hline 25 to $<30$ & 1.48 & $0.14-16.18$ & $0.749^{a)}$ \\
\hline$>30$ & 1.00 & & \\
\hline \multicolumn{4}{|l|}{ Tumor grade } \\
\hline $\mathrm{T} 1$ & 1.00 & & \\
\hline $\mathrm{T} 2$ & 4.97 & $1.06-23.25$ & $0.041^{\mathrm{a})}$ \\
\hline T3 & 3.18 & $0.42-24.18$ & $0.264^{a)}$ \\
\hline $\mathrm{T} 4$ & 18.34 & $0.66-512.45$ & $0.086^{a)}$ \\
\hline \multicolumn{4}{|l|}{ Lymph node } \\
\hline No & 1.00 & & \\
\hline N1 & 2.53 & $0.34-19.15$ & $0.368^{a)}$ \\
\hline N2 & 2.14 & $0.31-5.31$ & $0.447^{\mathrm{a})}$ \\
\hline N3 & 5.00 & $0.11-236.25$ & $0.413^{\mathrm{a})}$ \\
\hline \multicolumn{4}{|l|}{ Clinical staging } \\
\hline$\|$ & 1.00 & & \\
\hline III & 1.32 & $0.39-4.46$ & 0.65 \\
\hline \multicolumn{4}{|l|}{ ER state } \\
\hline Positive & 1.00 & & \\
\hline Negative & 1.12 & $0.32-3.87$ & 0.861 \\
\hline \multicolumn{4}{|l|}{ PR state } \\
\hline Positive & 1.80 & $0.54-6.01$ & 0.339 \\
\hline Negative & 1.00 & & \\
\hline \multicolumn{4}{|l|}{ HER-2 overexpression } \\
\hline Positive & 1.00 & & \\
\hline Negative & 2.35 & $0.70-7.92$ & 0.167 \\
\hline \multicolumn{4}{|l|}{ Biological type } \\
\hline Luminal A & 9.85 & $1.09-89.21$ & 0.042 \\
\hline Luminal B & 1.00 & & \\
\hline HER-2 enriched & 6.15 & $0.66-57.60$ & 0.111 \\
\hline Triple negative & 1.23 & $0.28-5.45$ & 0.784 \\
\hline \multicolumn{4}{|c|}{ Neoadjuvant chemotherapy regimen } \\
\hline Non-target regimen & 3.70 & $1.05-13.03$ & 0.041 \\
\hline Target regimen & 1.00 & & \\
\hline
\end{tabular}

(Continued to the next page)
Table 5. Continued

\begin{tabular}{|c|c|c|c|}
\hline \multirow{2}{*}{ Characteristics } & \multicolumn{2}{|c|}{ Simple logistic regression } & \multirow{2}{*}{ P-value } \\
\hline & OR & $95 \% \mathrm{Cl}$ & \\
\hline \multicolumn{4}{|c|}{ Reduction of PBL counts } \\
\hline$<755 \times 10^{6} / \mathrm{L}$ & 4.84 & $1.31-17.90$ & 0.018 \\
\hline$\geq 755 \times 10^{6} / \mathrm{L}$ & 1.00 & & \\
\hline \multicolumn{4}{|l|}{ A } \\
\hline Lymphocyte & 1.00 & $1.00-1.00$ & 0.594 \\
\hline Neutrophil & 1.01 & $0.95-1.08$ & 0.751 \\
\hline Platelet & 1.00 & $0.99-1.01$ & 0.915 \\
\hline \multicolumn{4}{|l|}{ B } \\
\hline Lymphocyte & 1.00 & $1.00-1.00$ & 0.876 \\
\hline Neutrophil & 0.99 & $0.97-1.02$ & 0.592 \\
\hline Platelet & 1.00 & $0.99-1.01$ & 0.637 \\
\hline \multicolumn{4}{|l|}{ C } \\
\hline Lymphocyte & 1.00 & $1.00-1.00$ & 0.029 \\
\hline Neutrophil & 1.01 & $0.98-1.05$ & 0.524 \\
\hline Platelet & 1.00 & $0.99-1.01$ & 0.58 \\
\hline \multicolumn{4}{|l|}{ B-A } \\
\hline Lymphocyte & 1.00 & $1.00-1.00$ & 0.679 \\
\hline Neutrophil & 0.99 & $0.97-1.02$ & 0.513 \\
\hline Platelet & 1.00 & $0.99-1.01$ & 0.705 \\
\hline \multicolumn{4}{|l|}{ C-A } \\
\hline Lymphocyte & 1.00 & $1.00-1.00$ & 0.028 \\
\hline Neutrophil & 1.01 & $0.98-1.04$ & 0.673 \\
\hline Platelet & 1.00 & $0.99-1.01$ & 0.477 \\
\hline
\end{tabular}

$\mathrm{OR}$, odds ratio; $\mathrm{Cl}$, confidence interval; $\mathrm{BMI}$, body mass index; $\mathrm{ER}$, estrogen receptor; $P R$, progesterone receptor; HER-2, human epidermal growth factor receptor; $P B L$, peripheral blood lymphocyte; $A$, before the initiation of neoadjuvant chemotherapy; $B$, after the 1st cycle of neoadjuvant chemotherapy; $C_{\text {, }}$ after the completion of neoadjuvant chemotherapy.

a) P-value of logistic regression with Firth method.

vival rates as good as with surgery, but resulted in unacceptably high locoregional recurrence rates in patients with a clinical complete CR [15]. In the current study, we suggested the possibility of PBL reduction as a complementary predictive factor.

PBL serves as an indicator of immune system function in cancer patients, and its count is associated with solid tumor prognosis $[9,10,16]$. Additionally, it may have a role in anti-neoplastic effects and protecting the host against tumor development through an apoptosis-induced T-cell immune response $[17,18]$. In general, neutrophil levels are used as an index to determine a patient's immune status. However, the correlation between neutrophil levels and pathologic CR was not statistically significant in the current study.

Most chemotherapy drugs cause lymphocytes reduction which in turn can lead to lymphocytopenia, potentially damaging the effectiveness of the anti-tumor immune response. There are several 
Table 6. Multivariable analysis for predictive factors of pathologic complete response

\begin{tabular}{|c|c|c|c|c|c|c|}
\hline \multirow[t]{2}{*}{ Characteristics } & \multicolumn{3}{|c|}{$\begin{array}{l}\text { Simple logistic regression } \\
\text { (Ref. pathologic CR negative) }\end{array}$} & \multicolumn{3}{|c|}{$\begin{array}{l}\text { Multiple logistic regressiona) } \\
\text { (Ref. pathologic CR negative) }\end{array}$} \\
\hline & OR & $95 \% \mathrm{Cl}$ & P-value & $\mathrm{OR}$ & $95 \% \mathrm{Cl}$ & P-value \\
\hline \multicolumn{7}{|c|}{ Neoadjuvant chemotherapy regimen } \\
\hline Non-target regimen & 3.70 & $1.05-13.03$ & 0.041 & 37.86 & 3.63-394.59 & 0.002 \\
\hline Target regimen & 1.00 & & & 1.00 & & \\
\hline \multicolumn{7}{|l|}{ Reduction of PBL counts } \\
\hline$<755 \times 10^{6} / \mathrm{L}$ & 4.84 & $1.31-17.90$ & 0.018 & 7.12 & $1.36-37.29$ & 0.020 \\
\hline$\geq 755 \times 10^{6} / \mathrm{L}$ & 1.00 & & & 1.00 & & \\
\hline
\end{tabular}

Ref., reference; $\mathrm{CR}$, complete response; $\mathrm{OR}$, odds ratio; $\mathrm{Cl}$, confidence interval; $\mathrm{PBL}$, peripheral blood lymphocyte.

a) P-value of logistic regression with Firth method, stepwise selection.

studies on the predictive value of lymphocyte reduction during chemotherapy on DFS or OS [2,3]. Some reports have suggested that lymphocyte reduction during chemotherapy paradoxically has anti-tumor benefits by impairing regulatory T cells [19].

Less is known about the relevance of lymphocyte count with efficacy of NAC. Most clinical series have focused solely on the absolute count of lymphocytes, and factors predicting the response to chemotherapy have yet to be fully elucidated. Herein we evaluated the prognostic value of PBL changes and analyzed the association between decreased PBL count and pathologic CR in a group of breast cancer patients (stage II-III) treated with NAC.

In the present study, we retrospectively calculated the results of tests blood of 61 patients diagnosed with breast cancer and treated with NAC. We observed that the low reduction of PBL counts after the completion of NAC was relevant to pathologic CR regardless of baseline PBL count. The changes in absolute level of PBL had more meaningful results than the reduction ratio, suggesting its role as a marker for predicting a response to NAC. Similar to previous reports, results of the current study suggest that a good response to NAC correlates with both the tumor phenotype, such as small tumor size, and a treatment regimen that includes trastuzumab [20]. Recently, Vicente Conesa et al. [8] reported that both a low baseline PBL count and a large decrease of PBL after chemotherapy confer a worse prognosis for breast cancer recurrence. Similarly, baseline lymphocytopenia is found to be a strong independent prognostic factor for DFS and OS.

The results of the current study are further supported by finding that lymphocytes particularly cytotoxic $\mathrm{T}$ cells promote apoptosis and inhibit tumor growth. Notwithstanding toxicity of the chemotherapy regimens, maintaining lymphocytes can be beneficial for an anti-tumor effect during NAC. Therefore, a high number of PBL with anti-tumor activity could be an indicator of tumor suppression activity, especially for CD8 T cells [21]. This concept is further supported by the results of an improved pathologic CR af- ter NAC when a lymphocytic infiltrate is present in breast specimens from a pathological point of view [12].

Thus, these data suggest that low reduction of PBL might be related to anti-tumor activity, and better NAC response. Regarding decreases in PBL after the entire chemotherapy treatment, we do not have a clear explanation for its lack of prognostic value, though we speculate that the maintenance of PBL count in most patients could have an influence in not only the response to NAC but also OS or DFS.

There were some limitations to the current study. The potential effect of supportive treatment with immune system stimulation on PBL counts could not be completely ruled out. Chemotherapy agents induce bone marrow suppression and extravasation of neutrophils lead to neutropenia in the blood [22]. In addition, patients with low neutrophil counts are administered granulocyte macrophage-colony stimulating factor (GM-CSF) or granulocyte-colony stimulating factor (G-CSF) which stimulates granulocyte production. Particularly, G-CSF such as filgrastim, lenograstim, pegfilgrastim, is used to prevent or treat febrile neutropenia [23]. During 2-week courses of G-CSF, using G-CSF doses of $\geq 10 \mu \mathrm{g} / \mathrm{kg}$ of body weight per day increases neutrophil with monocyte and lymphocyte slightly [24]. In the current study, 25 of 61 patients (40.9\%) received GM-CSF, five received pegfilgrastim (Neulasta), and 20 received filgrastim (Grasin). The majority of patients who received doxorubicin and cyclophosphamide (AC) regimen were administered G-CSF for therapeutic purposes, except three patients who received Neulasta for prevention. Three (21\%) patients in achieving pathologic CR group and less than half of the patients (47\%) in non-achieving pathologic CR group were administered G-CSF. Recently, the National Comprehensive Cancer Network and the American Society of Clinical Oncology recommended the use of G-CSF prophylactically according to the risk of neutropenia [23].

Although almost all patients received a sequential combination of anthracyclines and taxanes, other schedules and chemotherapy 
regimens were also used, with potential differences in immunity impairment. For example, combination regimens of anthracyclines and taxanes can cause immunosuppression and lymphocytopenia. Furthermore, in the group using trastuzumab, pathologic $\mathrm{CR}$ tends to be better due to the targeting effect. These differences impair homogeneity of the study population and could result in some bias.

To understand the correlation between host immunity and anticancer therapeutic effect, it is necessary to evaluate not only lymphocyte number but also function. Therefore, a prospective clinical trial based on interferon-gamma secretion assay is needed to analyze the function of lymphocytes such as CD4+, CD8+ T cells, or NK cells.

Additional study with a large cohort sample size as well as with a more strictly controlled chemotherapy regimen and biological subtype variables would serve to validate the current findings. Additionally, long-term follow-up studies are needed to determine if associations exist between lymphocytes and long-term outcomes such as DFS and OS in breast cancer patients.

We concluded that PBL reduction during NAC might serve as a potential marker in expecting pathologic CR in localized breast cancer patients. The less PBL counts are reduced, the more favorable the outcomes. While our findings emphasize that low reduction of PBL counts alone is not sufficient to reliably rule out residual tumor, it nevertheless remains a potentiality. These findings support the need for further prospective studies with larger cohort and additional investigation of chemotherapy-induced lymphocyte reduction. In our future study, we will investigate the other complementary factors in predicting pathologic CR.

\section{CONFLICT OF INTEREST}

No potential conflict of interest relevant to this article was reported.

\section{REFERENCES}

1. Untch M, Konecny GE, Paepke S, von Minckwitz G. Current and future role of neoadjuvant therapy for breast cancer. Breast 2014;23:526-37.

2. Kong X, Moran MS, Zhang N, Haffty B, Yang Q. Meta-analysis confirms achieving pathological complete response after neoadjuvant chemotherapy predicts favourable prognosis for breast cancer patients. Eur J Cancer 2011;47:2084-90.

3. Ethier JL, Desautels D, Templeton A, Shah PS, Amir E. Prognostic role of neutrophil-to-lymphocyte ratio in breast cancer: a systematic review and meta-analysis. Breast Cancer Res 2017;19:2.
4. Croshaw R, Shapiro-Wright H, Svensson E, Erb K, Julian T. Accuracy of clinical examination, digital mammogram, ultrasound, and MRI in determining postneoadjuvant pathologic tumor response in operable breast cancer patients. Ann Surg Oncol 2011;18:31603.

5. Gazet JC, Coombes RC, Ford HT, Griffin M, Corbishley C, Makinde $\mathrm{V}$, et al. Assesssment of the effect of pretreatment with neoadjuvant therapy on primary breast cancer. Br J Cancer 1996;73: 758-62.

6. Fisher CS, Ma CX, Gillanders WE, Aft RL, Eberlein TJ, Gao F, et al. Neoadjuvant chemotherapy is associated with improved survival compared with adjuvant chemotherapy in patients with triple-negative breast cancer only after complete pathologic response. Ann Surg Oncol 2012;19:253-8.

7. Rouzier R, Perou CM, Symmans WF, Ibrahim N, Cristofanilli M, Anderson K, et al. Breast cancer molecular subtypes respond differently to preoperative chemotherapy. Clin Cancer Res 2005;11: 5678-85.

8. Vicente Conesa MA, Garcia-Martinez E, Gonzalez Billalabeitia E, Chaves Benito A, Garcia Garcia T, Vicente Garcia V, et al. Predictive value of peripheral blood lymphocyte count in breast cancer patients treated with primary chemotherapy. Breast 2012;21:46874.

9. Kitayama J, Yasuda K, Kawai K, Sunami E, Nagawa H. Circulating lymphocyte number has a positive association with tumor response in neoadjuvant chemoradiotherapy for advanced rectal cancer. Radiat Oncol 2010;5:47.

10. Li Y, Wei Y, He Q, Wang X, Fan C, Li G. Clinicopathological and prognostic significance of high circulating lymphocyte ratio in patients receiving neoadjuvant chemotherapy for advanced gastric cancer. Sci Rep 2018;8:6223.

11. Asano Y, Kashiwagi S, Onoda N, Noda S, Kawajiri H, Takashima T, et al. Platelet-lymphocyte ratio as a useful predictor of the therapeutic effect of neoadjuvant chemotherapy in breast cancer. PLoS One 2016;11:e0153459.

12. Wang K, Xu J, Zhang T, Xue D. Tumor-infiltrating lymphocytes in breast cancer predict the response to chemotherapy and survival outcome: a meta-analysis. Oncotarget 2016;7:44288-98.

13. Graziano V, Grassadonia A, Iezzi L, Vici P, Pizzuti L, Barba M, et al. Combination of peripheral neutrophil-to-lymphocyte ratio and platelet-to-lymphocyte ratio is predictive of pathological complete response after neoadjuvant chemotherapy in breast cancer patients. Breast 2019;44:33-8.

14. American Joint Committee on Cancer. Cancer staging manual breast cancer. 8th ed. Chicago, IL: American Joint Committee on Cancer; 2017.

15. Ring A, Webb A, Ashley S, Allum WH, Ebbs S, Gui G, et al. Is sur- 
gery necessary after complete clinical remission following neoadjuvant chemotherapy for early breast cancer? J Clin Oncol 2003;21: 4540-5.

16. Fogar P, Sperti C, Basso D, Sanzari MC, Greco E, Davoli C, et al. Decreased total lymphocyte counts in pancreatic cancer: an index of adverse outcome. Pancreas 2006;32:22-8.

17. DeNardo DG, Coussens LM. Inflammation and breast cancer. Balancing immune response: crosstalk between adaptive and innate immune cells during breast cancer progression. Breast Cancer Res 2007;9:212.

18. Zitvogel L, Apetoh L, Ghiringhelli F, Andre F, Tesniere A, Kroemer G. The anticancer immune response: indispensable for therapeutic success? J Clin Invest 2008;118:1991-2001.

19. Zhang L, Dermawan KT, Jin ML, Xiong SD, Chu YW. Does chemotherapy augment anti-tumor immunotherapy by preferential impairment of regulatory T cells? Med Hypotheses 2008;71:802-4.

20. Untch M, Fasching PA, Konecny GE, Hasmuller S, Lebeau A, Kreienberg R, et al. Pathologic complete response after neoadjuvant chemotherapy plus trastuzumab predicts favorable survival in human epidermal growth factor receptor 2-overexpressing breast cancer: results from the TECHNO trial of the AGO and GBG study groups. J Clin Oncol 2011;29:3351-7.

21. Menard C, Martin F, Apetoh L, Bouyer F, Ghiringhelli F. Cancer chemotherapy: not only a direct cytotoxic effect, but also an adjuvant for antitumor immunity. Cancer Immunol Immunother 2008;57:1579-87.

22. Kuderer NM, Dale DC, Crawford J, Cosler LE, Lyman GH. Mortality, morbidity, and cost associated with febrile neutropenia in adult cancer patients. Cancer 2006;106:2258-66.

23. Smith TJ, Bohlke K, Lyman GH, Carson KR, Crawford J, Cross SJ, et al. Recommendations for the use of WBC growth factors: American Society of Clinical Oncology clinical practice guideline update. J Clin Oncol 2015;33:3199-212.

24. Lieschke GJ, Burgess AW. Granulocyte colony-stimulating factor and granulocyte-macrophage colony-stimulating factor (1). N Engl J Med 1992;327:28-35. 